\title{
Severity One Out of Three
}

National Cancer Institute

\section{Source}

National Cancer Institute. Severity One Out of Three. NCI Thesaurus. Code C147499.

A severity result of one, on a three level scale. 